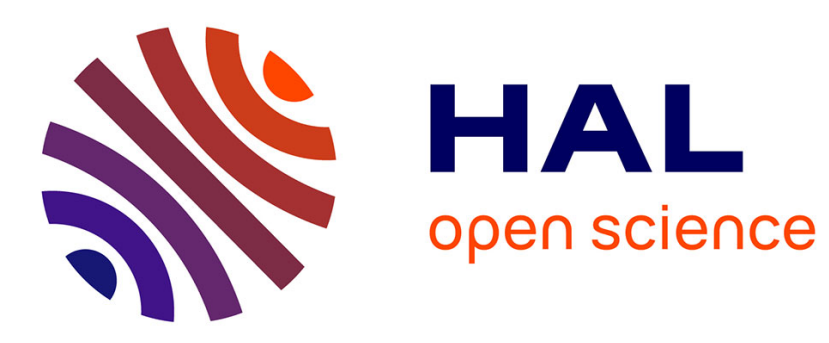

\title{
Tilting a Ground State Reactivity Landscape by Vibrational Strong Coupling
}

Anoop Thomas, Thibault Chervy, Lucas Lethuillier-Karl, Kalaivanan Nagarajan, Robrecht M A Vergauwe, Jino George, Atef Shalabney, Éloïse Devaux, Cyriaque Genet, Joseph Moran, et al.

\section{To cite this version:}

Anoop Thomas, Thibault Chervy, Lucas Lethuillier-Karl, Kalaivanan Nagarajan, Robrecht M A Vergauwe, et al.. Tilting a Ground State Reactivity Landscape by Vibrational Strong Coupling. Science, 2019, 363 (6427), pp.615-619. 10.26434/chemrxiv.7160789.v1 . hal-02413303

\section{HAL Id: hal-02413303 https://hal.science/hal-02413303}

Submitted on 16 Dec 2019

HAL is a multi-disciplinary open access archive for the deposit and dissemination of scientific research documents, whether they are published or not. The documents may come from teaching and research institutions in France or abroad, or from public or private research centers.
L'archive ouverte pluridisciplinaire HAL, est destinée au dépôt et à la diffusion de documents scientifiques de niveau recherche, publiés ou non, émanant des établissements d'enseignement et de recherche français ou étrangers, des laboratoires publics ou privés. 


\section{Tilting a Ground State Reactivity Landscape by Vibrational Strong Coupling}

Anoop Thomas, Lucas Lethuillier-Karl, Kalaivanan Nagarajan, Robrecht M. A. Vergauwe, Jino George, Thibault Chervy, Atef Shalabney, Eloïse Devaux, Cyriaque Genet, Joseph Moran, Thomas W. Ebbesen Submitted date: 03/10/2018 - Posted date: 04/10/2018

Licence: CC BY-NC-ND 4.0

Citation information: Thomas, Anoop; Lethuillier-Karl, Lucas; Nagarajan, Kalaivanan; M. A. Vergauwe, Robrecht; George, Jino; Chervy, Thibault; et al. (2018): Tilting a Ground State Reactivity Landscape by Vibrational Strong Coupling. ChemRxiv. Preprint.

Site-selectivity is fundamental for steering chemical reactivity towards a given product and various efficient chemical methods have been developed for this purpose. Here we explore a very different approach by using vibrational strong coupling (VSC) between a reactant and the vacuum field of a microfluidic optical cavity. For this purpose, the reactivity of a compound bearing two possible silyl bond cleavage sites, at $\mathrm{Si}-\mathrm{C}$ and $\mathrm{Si}-\mathrm{O}$, was studied as a function of VSC of its various vibrational modes in the dark. The results show that VSC can indeed tilt the reactivity landscape to favor one product over the other. Thermodynamic parameters reveal the presence of a large activation barrier and significant changes to the activation entropy, confirming the modified chemical landscape under strong coupling. This study shows for the first time that VSC can impart site-selectivity for chemical reactions without the need for chemical intervention.

File list (2)

Site Selectivity MS ChemRxiv.pdf (1.49 MiB)

view on ChemRxiv • download file

Site Selectivity SI ChemRxiv.pdf (1.44 MiB)

view on ChemRxiv • download file 


\section{Tilting a Ground State Reactivity Landscape by Vibrational Strong Coupling}

A. Thomas ${ }^{1 \#}$, L. Lethuillier-Karl ${ }^{1 \#}$, K. Nagarajan ${ }^{1}$, R. M. A. Vergauwe ${ }^{1}$, J. George ${ }^{1 \ddagger}$, T. Chervy $^{1 \dagger}$, A. Shalabney ${ }^{2}$, E. Devaux ${ }^{1}$, C. Genet ${ }^{1}$, J. Moran $^{1 *}$, T. W. Ebbesen ${ }^{1 *}$

${ }^{1}$ University of Strasbourg, CNRS, ISIS \& icFRC, 8 allée G. Monge, 67000 Strasbourg, France.

${ }^{2}$ Braude College, Snunit Street 51, Karmiel 2161002, Israel

*Correspondence to: ebbesen@unistra.fr; moran@unistra.fr

\#These authors contributed equally

Present Address:

Department of Chemistry. Indian Institute of Science Education and research Mohali, Mohali, India.

†Department of Physics, ETH Zurich, Switzerland

\section{Abstract:}

Site-selectivity is fundamental for steering chemical reactivity towards a given product and various efficient chemical methods have been developed for this purpose. Here we explore a very different approach by using vibrational strong coupling (VSC) between a reactant and the vacuum field of a microfluidic optical cavity. For this purpose, the reactivity of a compound bearing two possible silyl bond cleavage sites, at $\mathrm{Si}-\mathrm{C}$ and $\mathrm{Si}-\mathrm{O}$, was studied as a function of VSC of its various vibrational modes in the dark. The results show that VSC can indeed tilt the reactivity landscape to favor one product over the other. Thermodynamic parameters reveal the presence of a large activation barrier and significant changes to the activation entropy, confirming the modified chemical landscape under strong coupling. This study shows for the first time that VSC can impart site-selectivity for chemical reactions without the need for chemical intervention.

Over the years, synthetic chemists have mastered the art of designing and developing bond-, siteand stereo-selective chemical reactions using various functional moieties and catalysts (1-4). In light of the decisive nature of molecular vibrations in dictating the outcome of isomerization and other chemical processes (5-7), a physical approach based on the selective excitation of vibrational modes using strong laser fields was also developed to impart control on chemical reactivity $(8-11)$. This laser-induced mode-selective chemistry provided a way to move the system along the reaction coordinate, particularly for late barrier reactions (12-14). Interestingly, the vibrational excitation of the $\mathrm{C}-\mathrm{H}$ bond of an early barrier reaction inhibited the formation of the typical product and modified the branching ratio (15), even though such an excitation was predicted to have little influence on the outcome (16). An adiabatic transfer of excitation to the product stretching mode was also observed when the localized spectator mode of the reactant was pumped in the former experiments, further indicating the complexities of the reactive landscape $(15,17)$. Although such mode-selective chemistry works well in the gas phase at cryogenic temperatures, intramolecular vibrational energy redistribution (IVR) limits its use for 
reactions in solution involving large molecules. To overcome such challenges, we started exploring an alternative approach whereby the vibrational transition of a molecule is selectively hybridized with the vacuum electromagnetic field (zero-point energy) of a cavity by means of vibrational strong coupling (VSC) (18-21). VSC leads to splitting of the vibrational transition into two new vibropolaritonic states $(\mathrm{P}+$ and $\mathrm{P}-$ in Fig. 1A) that should affect the Morse potential. We predicted that this could lead to changes in the chemical reactivity and indeed in a first example, we demonstrated that the rate of silyl cleavage is reduced by the VSC of the Si-C stretching vibration (22). Most significantly, the reaction mechanism was also modified.

Changing the ground state reactivity landscape under such a weak field perturbation suggests that VSC could also be used for site-selectivity of a reaction. Here in a proof-ofprinciple study, we demonstrate that it is indeed possible to tilt the chemical landscape of a reactant to favor one product over another.
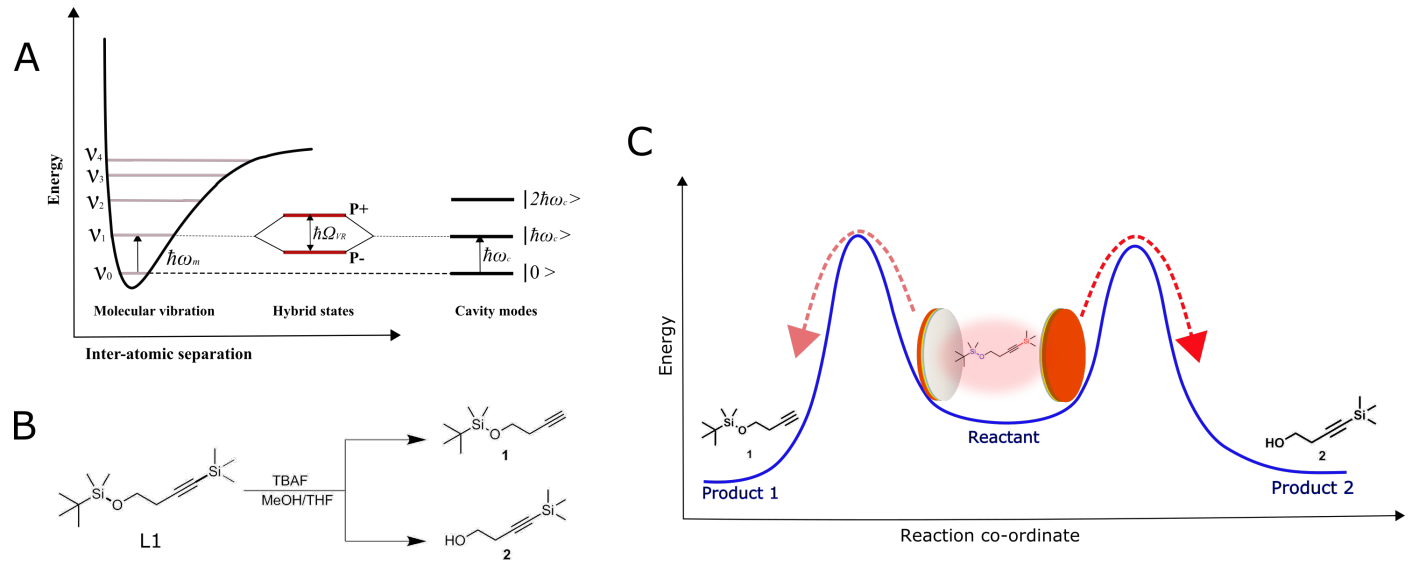

Fig. 1. (A) Schematic illustration of the light-matter strong coupling of a vibrational transition in resonance with Fabry-Pérot cavity mode and the subsequent formation of hybrid polaritonic states $\mathrm{P}+$ and $\mathrm{P}$ - separated by Rabi splitting energy, $\hbar \Omega_{\mathrm{R}}$. (B) The scheme showing the two major silyl cleavage pathways for the starting material L1, when reacted with TBAF in a mixture of methanol and THF. The structure of the $C$ - (1) and $O$-deprotected product (2) are also given. (C) Schematic representation of a site selective reactive pathway upon the VSC of the reactant.

To explore the above possibilities, the silane derivative, tert-butyldimethyl((4(trimethylsilyl)but-3-yn-1-yl)oxy)silane called L1 hereafter, (structure given in Fig.1B), having two parallel silyl bond cleavage sites was synthesized (Supplementary Material for details). The nucleophilic attack of tetra- $n$-butylammonium fluoride (TBAF) on the Si atoms of L1 can result in the cleavage of the $\mathrm{Si}-\mathrm{C}$ bond, yielding product 1, or the breakage of the $\mathrm{Si}-\mathrm{O}$ bond, resulting in product 2 (Fig.1B). Since both the products are formed through similar mechanistic pathways, first we explored whether the selective strong coupling of vibrational modes associated to Si-C and Si-O have similar or different influences on the reactivity. The second and most important point of the present study was to check whether changes in the reactivity landscape under VSC lead to site-selectivity and thereby in changes to the branching ratio of the products as schematically presented in Fig.1B and C. 
It is recalled that light-matter hybridization is generally achieved by placing a molecule in liquid or solid form in a Fabry-Pérot optical cavity with a mode tuned in resonance with the chosen molecular transition (23). When the exchange of energy between the molecular transition and cavity mode is faster than any decay process, the system is said to be in a strong coupling regime manifested by the splitting of the molecular transition into two new states separated by a Rabi splitting energy, $\hbar \Omega_{\mathrm{R}}$, as shown schematically in Fig. 1A with VSC as a specific example. Under collective strong coupling conditions, the Rabi splitting can be so large that it results in significant modification of characteristic molecular and material properties beyond chemical reactivity such as work function (24), phase transition (25) and other physical processes including electrical conductivity (26), energy transfer (27-29) and reverse intersystem crossing (30).

The Fabry-Pérot cavity used in the present experiments was made of two parallel metal mirrors (Au coated $\mathrm{ZnSe}$ ) separated from each other by a Mylar spacer (6-8 um) having a hollow central channel designed to inject liquid samples (19). To ensure that the reaction dynamics are not affected by the presence of $\mathrm{Au}$ or $\mathrm{ZnSe}$, an additional layer of $200 \mathrm{~nm}$ glass $\left(\mathrm{SiO}_{2}\right)$ was deposited on top of the metal coating in each of the windows. A homogenous solution of the starting material L1 $(0.90 \mathrm{M})$ and TBAF $(0.86 \mathrm{M})$ in a $1: 1(\mathrm{v} / \mathrm{v})$ mixture of methanol and THF, optimized for strong coupling conditions, was used as the reacting medium throughout the experiments presented here. The IR spectrum of L1 (Fig. 2A) in this mixture recorded outside the cavity (a sample cell made of glass coated $\mathrm{ZnSe}$ windows separated by the same spacer thickness as of the Fabry-Pérot cavity) shows the characteristic strong bands corresponding to the stretching mode of Si-C $\left(842 \mathrm{~cm}^{-1}\right)$ and $\mathrm{Si}-\mathrm{O}\left(1110 \mathrm{~cm}^{-1}\right)$ and the bending mode of $\mathrm{CH}_{3}$ group bonded to $\mathrm{Si}\left(1250 \mathrm{~cm}^{-1}\right)$. 

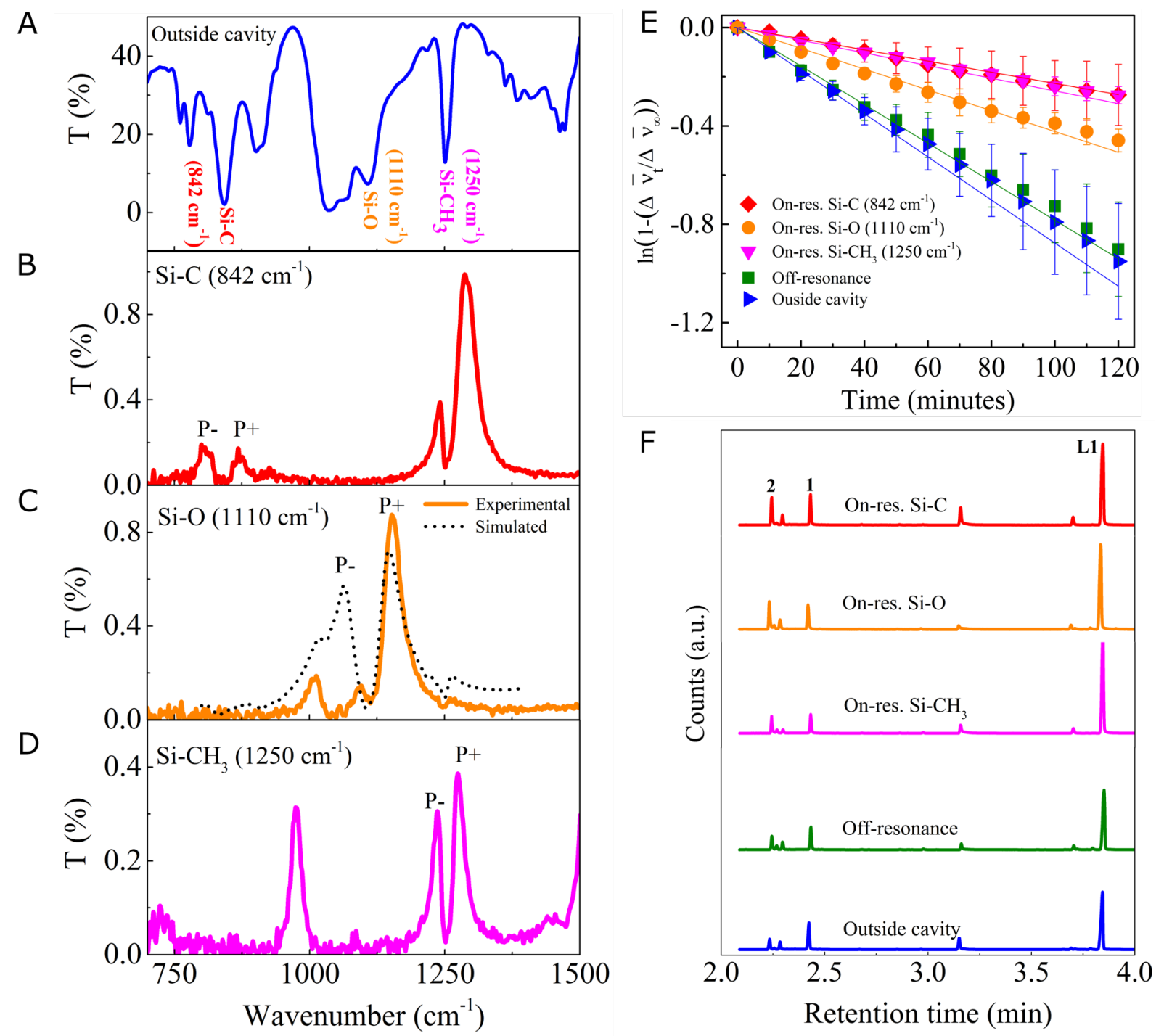

Fig. 2. (A) FT-IR transmission spectrum of the starting material L1 in the reaction mixture recorded outside the cavity. The characteristic peaks of L1 are noted in the plot. (B-D) The FTIR transmission spectra showing the vibrational strong coupling with the formation of $\mathrm{P}+$ and $\mathrm{P}-$ when the three different modes are coupled to the cavity: the Si-C stretching mode in (B), the Si$\mathrm{O}$ stretching in $(\mathrm{C})$ together with a transfer matrix simulated transmission spectrum shown by black dotted line, and (D) $\mathrm{Si}_{-} \mathrm{CH}_{3}$ bending vibration. (E) Kinetics of the reactions in cavities tuned to be on-resonance with the Si-C stretching (red diamonds), the Si-O stretching (orange circles) and the $\mathrm{Si}_{-} \mathrm{CH}_{3}$ bending modes (magenta triangles) together with an off-resonance cavity (green squares) and outside the cavity (blue triangles), as extracted from the temporal shifts in the higher-order cavity modes. (F) The GC-MS chromatograms of the reactions carried out in cavities tuned to be on-resonance with Si-C stretching (red), Si-O stretching (orange) and $\mathrm{Si}^{-\mathrm{CH}_{3}}$ bending modes (magenta), together with the data for an off-resonance cavity (green) and outside the cavity (blue). The unreacted starting material peak is marked as L1 and the peak corresponding to $\mathrm{C}$ - and $\mathrm{O}$ - deprotected products are marked as 1 and 2, respectively. The small peak at a retention time of 3.2 min corresponds to the unreacted TBAF. 
For VSC, it is important to tune the cavity mode to be in resonance with a specific IR transition of L1. This is achieved by carefully adjusting the spacer thickness by applying a pressure by tightening or loosening the screws holding the assembly (Supplementary Materials for details). The injection of the homogeneous reaction mixture into the flow cell Fabry-Pérot cavity, whose second mode $(\lambda)$ was precisely tuned to $842 \mathrm{~cm}^{-1}$, resulted in the strong coupling of the Si-C stretching transition as shown in Fig. 2B. The appearance of $\mathrm{P}+$ and $\mathrm{P}-$ peaks in the IR spectrum with a Rabi splitting energy of $70 \mathrm{~cm}^{-1}$, larger than the width of the cavity mode (30 $\left.\mathrm{cm}^{-1}\right)$ and the Si-C vibrational band $\left(30 \mathrm{~cm}^{-1}\right)$, showed that the system meets the strong coupling criteria (23). Under these conditions, a largely detuned coupling was also observed for the bending mode of $-\mathrm{CH}_{3}$ groups bonded to $\mathrm{Si}\left(\mathrm{Si}-\mathrm{CH}_{3}, 1250 \mathrm{~cm}^{-1}\right)$ to the third mode of the cavity. The role of strongly coupled $\mathrm{Si}-\mathrm{CH}_{3}$ bending vibration towards the reactivity was analyzed separately and is described later. In a different experiment, by tuning the third mode of the cavity to $1110 \mathrm{~cm}^{-1}$, VSC of Si-O stretching band was achieved (Fig. 2C solid line). The presence of the intense and broad vibrational modes of solvents (1040-1060 $\left.\mathrm{cm}^{-1}\right)$, splits and reduces the P-band intensity (Fig. 2C) and complicates the spectrum analysis. Therefore the Rabi splitting energy in this case was calculated using the standard optics technique (transfer matrix simulation) and shown in Fig. $2 \mathrm{C}$ as a dotted line. The extracted value of $85 \mathrm{~cm}^{-1}$ is well above the cavity and IR mode widths.

The rate of silyl cleavage of L1 under the influence of VSC as well as the product ratio between 1 and 2 was quantitatively estimated using spectroscopic and chromatographic methods respectively. The sum rate of the two parallel reactions, carried out at room temperature (298 K), was determined from the $\mathrm{ln}$ plot of the temporal shift of a higher order cavity mode (from $\bar{v}_{0}$ to $\bar{v}_{\mathrm{t}}$; see Supplementary Materials for details). Similar to the earlier experiment (22), this was aided by the slight change in the refractive indices of the reactant and the products of the reaction. The linear $\ln$ plot (Fig. 2E) indicated that the kinetics is first order and from the slope the rate constant corresponding to the disappearance of starting material was determined. Interestingly, when the Si-C stretching bond vibration was strongly coupled, the rate was retarded (Fig. 2E, red diamond) by a factor of 3.5 compared to the control measurements done outside the cavity (Fig. 2E, blue triangle) or inside the cavity under off-resonance conditions (Fig. 2E, green square). Off-resonant experiments correspond to the set of measurements done in the Fabry-Pérot cavity, where the modes are not in resonance with any of the selected vibrational transitions of L1.

To better understand the reactivity and product formation, the reaction mixture was quantitatively analyzed by GC-MS after each experiment (see Supplementary Materials for details). The presence of the unreacted starting material peak (denoted as L1 in Fig. 2F) in the chromatogram of all the experiments shows that the reaction has not gone to completion in the 3 $\mathrm{h}$ time period. The ratio between the concentration ([1]/[2]) of the products 1 and 2 of Fig. 1B was further quantified as shown in Fig. 2F. For outside cavity and off-resonance experiments, the ratio ([1]/[2]) between the $\mathrm{C}$ - (1) and $\mathrm{O}$ - (2) deprotected products was found to be $1.5 \pm 0.2$ and $1.4 \pm 0.1$ respectively. A remarkable increase in the peak area of product 2 was seen when the $\mathrm{Si}$ $\mathrm{C}$ stretching vibration was strongly coupled (red chromatogram, Fig. 2F), changing the branching ratio [1]/[2] to $0.3 \pm 0.1$. This value takes into account the fraction of the Fabry-Perot microfluidic cavity under strong coupling. This reversal of product ratio under VSC indicates that the reactive landscape is modified in favor of $\mathrm{Si}-\mathrm{O}$ cleavage rather than the typical 
kinetically favored Si-C bond breaking. A similar trend was observed when the Si-O stretching vibration was also subjected to light-matter hybridization. In this case where the overall rate of the reaction was slowed by a factor of 2.5 relative to the outside cavity/off resonant conditions (orange circle, Fig. $2 \mathrm{E}$ ), the product ratio was estimated as $0.4 \pm 0.1$. Thus, the VSC of the two stretching vibrations of the $\mathrm{Si}$ reactive center both tilt the reaction towards $\mathrm{Si}-\mathrm{O}$ bond cleavage, clearly imparting selectivity.

As discussed in the introduction, previous examples on the mode-selective chemistry experiments have shown that spectator modes, vibrations that are not directly linked to the reaction coordinate, also play a critical role in determining the outcome of reactions $(14,15,31)$. In the present case, the strong IR band at $1250 \mathrm{~cm}^{-1}$, arising from the bending mode of the $-\mathrm{CH}_{3}$ groups attached to the Si atom (32) may also be considered a spectator vibration. However, the appearance of this IR transition at $1250 \mathrm{~cm}^{-1}$, which in isolation should be at ca. $1460 \mathrm{~cm}^{-1}$, already indicates that it is interacting with other vibrations associated with the $\mathrm{Si}$ center. By varying the spacer thickness, VSC of this bending mode alone (Fig. 2D) was achieved with a Rabi splitting energy of $43 \mathrm{~cm}^{-1}$, without simultaneously coupling either the Si-C or Si-O stretching vibrations. The VSC of the $1250 \mathrm{~cm}^{-1}$ reduced the overall reaction rate by a factor of 3.5 (Fig. 2E, magenta triangle) with a ratio [1]/[2] of $0.3 \pm 0.1$ between the products (Fig. 2F, magenta chromatogram), in a close similarity to the results obtained under the VSC of the Si-C stretching transition. A question which then naturally arises is whether the VSC of any vibrational mode of L1 would lead to similar results or whether the effects are only related to those vibrations that are associated with the Si reactive center. To check this, the C-O stretching transition (at $1045 \mathrm{~cm}^{-1}$ ) of $\mathrm{L} 1$, a vibration that should have little influence on the reaction from a mechanistic point of view, was chosen. Moreover, this mode is embedded in the broad region corresponding to the C-O vibration (1040-1060 $\mathrm{cm}^{-1}$, see Fig. 2A) of the solvents used (methanol and THF). Therefore, the VSC of the $\mathrm{C}-\mathrm{O}$ transition should also provide information on whether coupling a solvent vibration influences the outcome of this chemical reaction. Interestingly, the rate as well as the ratio of the product concentration was not affected by the VSC of the C-O transition (Fig. S6). It shows that in the present experiment only those vibrations that are directly associated with the Si reaction center are influential in tilting the reaction landscape as one might expect. Although VSC of the C-O vibration of methanol did not influence the present study, VSC of solvent could potentially lead to significant changes for reactions in which solvent plays a more crucial role. Further, through a series of experiments where the cavity tunings were varied throughout the IR spectrum of L1, an action spectrum was obtained (Fig. 3A) which clearly shows through which vibrational modes VSC act to influence the reaction. This plot of the overall rate constant $\left(k_{T R}\right)$ as a function of cavity tuning reflects the precise selectivity that can be achieved by this weak field physical perturbation tool at room temperature.

Furthermore, by combining the observed overall rate constant $\left(k_{T R}\right)$ of the parallel reaction and the product ratios $([1] /[2])$ from GC-MS measurements, the individual rate constants corresponding to the Si-C $\left(k_{1}\right)$ and $\mathrm{Si}-\mathrm{O}\left(k_{2}\right)$ bond cleavage were estimated. From this data, the respective product yields were calculated. The yields $(\phi)$ of products 1 and 2 in and out of strong coupling for various coupling conditions are shown in Fig. 3B. Outside the cavity and in off-resonance conditions, the $k_{1}$ dominates over $k_{2}$ resulting in preferential formation of product 1 (Fig. 3B, violet diamond). Interestingly, the VSC reverses the selectivity, leading to an excess formation of product 2 (Fig. 3B, pink squares). This is again a direct proof-of-principle that the chemical landscape can be tilted by targeting the vibrational modes associated with the 
reaction center. It should be noted that the value of both $k_{1}$ and $k_{2}$ under strong coupling conditions are both lower than those observed outside the cavity indicating that a higher activation energy is required for the reaction under VSC. It should be noted that products 1 and 2 undergo further silyl bond cleavage, however the subsequent reactions appear to be much slower resulting in the accumulation of 1 and 2 in the GC-MS analysis. Nevertheless, it imparts a small error in the yields but has no consequence on the overall results. As will be discussed further down, the change in yields under VSC are probably much higher if one takes into account physical parameters associated with the coupling process.

A

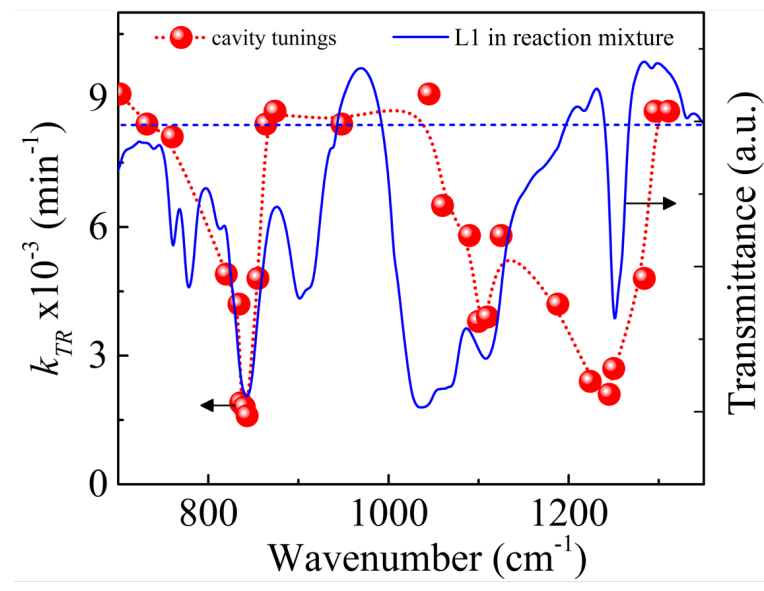

B

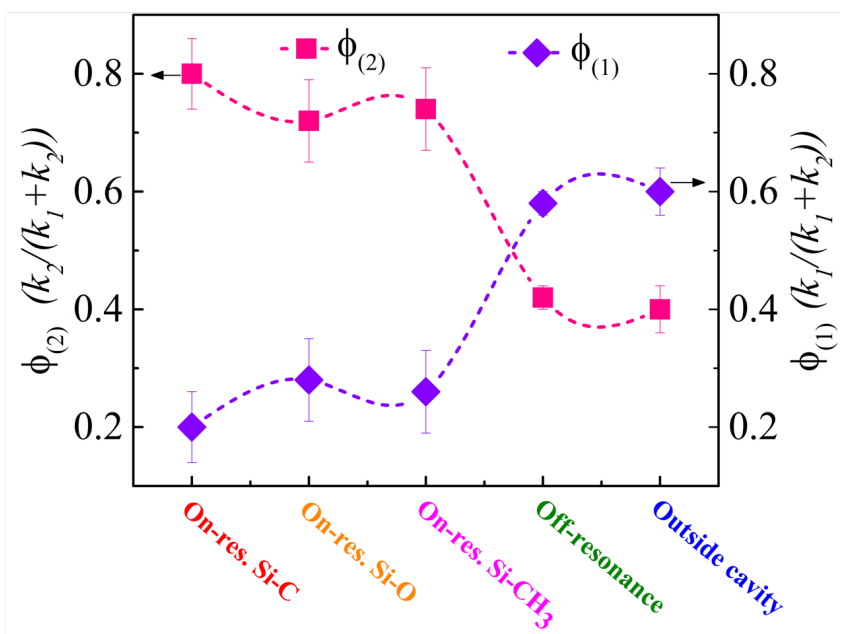

Fig. 3. (A) The overall reaction rate as a function of the cavity tuning for reactions inside the cavity (red spheres). The blue solid line shows the IR absorption spectrum of L1 in the reaction medium. The red dotted line connecting the spheres is a guide for the eye. The blue dashed line represents the average rate of the reaction outside the cavity. (B) Plot showing the yield of the product 1 ( $\phi_{1}$, violet diamonds) and 2 ( $\phi_{2}$, pink squares) under VSC of various vibrational modes of L1, together with the off-resonance and outside cavity conditions. The dashed lines are guides for the eye.

The thermodynamics of the reaction were determined by studying the reaction rate as a function of temperature under VSC by coupling of the Si-C and Si-O stretching vibrations and outside the cavity. By using transition state theory, the free energy of activation $\left(\Delta \mathrm{H}^{\ddagger}\right)$ and the entropy of activation $\left(\Delta \mathrm{S}^{\ddagger}\right)$ were determined from the slope and intercept of the plot of $\ln \left(k_{T R}\right)$ against the inverse of temperature. Interestingly, the $\Delta \mathrm{H}^{\ddagger}$ values under $\mathrm{VSC}, 66.8 \pm 3 \mathrm{kJmol}^{-1}$ (for $\mathrm{Si}-\mathrm{C}$ ) and $62.1 \pm 3 \mathrm{kJmol}^{-1}$ (for $\mathrm{Si}-\mathrm{O}$ ) were much higher than that observed for outside cavity $\left(27.2 \pm 3 \mathrm{kJmol}^{-1}\right)$ experiments. This high activation energy requirement reflects the observed retardation of the bond breaking process for the selected reaction under VSC. The $\Delta \mathrm{S}^{\ddagger}$ values were also distinctly different under VSC, $-66.5 \pm 11 \mathrm{Jmol}^{-1} \mathrm{~K}^{-1}$ for Si-C and $-80.6 \pm 11 \mathrm{Jmol}^{-1} \mathrm{~K}^{-1}$ for Si-O while outside the cavity it is found to be $-190.4 \pm 8 \mathrm{Jmol}^{-1} \mathrm{~K}^{-1}$. Since a typical fluoride induced silyl cleavage is known to proceed through a pentacoordinate intermediate, a strained transition state with large negative value for $\Delta \mathrm{S}^{\ddagger}$ is expected as observed outside the cavity. A 
sharp decrease of $\Delta \mathrm{S}^{\ddagger}$ to a lower value under VSC implies that the transition state under VSC is significantly different. These changes in the $\Delta \mathrm{H}^{\ddagger}$ and the $\Delta \mathrm{S}^{\ddagger}$ are indeed an indication that mechanism, and thereby the chemical landscape, changes under strong coupling conditions in agreement with our earlier study (22).

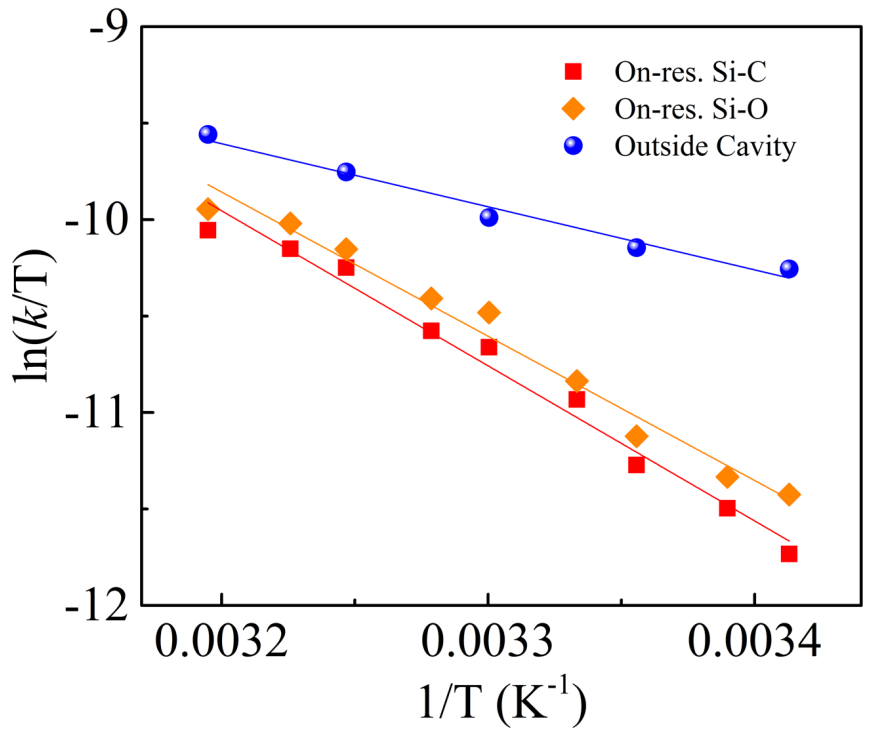

Fig. 4. Eyring plot showing the reaction rate as a function of temperature for reactions inside the cavities tuned to be on-resonance with $\mathrm{Si}-\mathrm{C}$ (red squares) and $\mathrm{Si}-\mathrm{O}$ (orange diamonds) stretching modes and outside the cavity (blue spheres).

The above results show that VSC can modify the chemical landscape in such a way that it tilts the landscape from one product to another. An interesting aspect of chemical reactions under VSC so far has been the high activation barrier seen towards the breaking of Si-C and Si-O bonds. A possible reason could be motional narrowing of the Morse curves in this collective coupling of many molecules to a given optical mode, akin to what occurs in J-aggregates (33), increasing the energy barrier. This barrier increase has also been seen in earlier experiments, whether occurring in the ground state (22) or via the excited state under electronic strong coupling such as in the photoisomerization reaction of merocyanine (34). For the latter case, recent theoretical studies show that the collective response of molecules plays a role in widening the barrier at the conical intersection and thereby preventing the isomerization $(35,36)$. More experiments and theoretical studies are required to determine whether such features can be generalized or not. The high activation energy and the less negative $\Delta \mathrm{S}$ clearly indicates that the reaction follows a dissociative mechanism under VSC, and also suggest that the transition state is product-like as in the case of late barrier reactions. Factors such as changes to the electrophilicity of the $\mathrm{Si}$ atom could be a reason for the enhanced rupture of the $\mathrm{Si}-\mathrm{O}$ bond under VSC.

In conclusion, this proof-of-principle study is a first step towards controlling reaction selectivity 25 at a given site through VSC. It is already clear that it requires that the VSC is applied to vibrational transitions directly linked to the reaction center, in this case the Si atom. It should be noted that strong coupling is dependent on the orientation of the transition dipole moment 
relative to the electric field of the cavity mode. Here the molecules were randomly oriented and the electric field is not uniform in the cavity, i.e. there are nodes where the field is essentially null. Hence, the five-fold increase in selectivity under VSC reported here is probably much larger if corrected for such features. For instance, experiments could be done in a tubular cavity to ensure a larger fraction of coupled molecules. Therefore, the selectivity and specificity displayed by VSC in the current experiment shows the remarkable potential of light-matter strong coupling for enhancing site selectivity of organic and inorganic chemical reactions. More reactions must be studied to evaluate the generality of this phenomenon and to see to what extent the selectivity can be enhanced at a given site. As more and more studies show (18-22, 37-49), this weak field method that works at room temperature has the potential to become an everyday tool for chemists to physically control chemical reactivity without catalysts, pre-functionalization or chemical changes to the reaction conditions.

\section{References}

1. A. H. Hoveyda, D. A. Evans, G. C. Fu, Chem. Rev. 93, 1307-1370 (1993).

2. N. A. Romero, K. A. Margrey, N. E. Tay, D. A. Nicewicz, Science. 349, 1326-1330 (2015).

3. Z. Huang, G. Dong, Acc. Chem. Res. 50, 465-471 (2017).

4. K. Godula, D. Sames, Science. 312, 67-72 (2006).

5. R. T. Hall, G. C. Pimentel, J. Chem. Phys. 38, 1889-1897 (1963).

6. Q. Wang, R. W. Schoenlein, L. A. Peteanu, R. A. Mathies, C. V. Shank, Science. 266, 422424 (1994).

7. M. Delor et al., Science. 346, 1492-1495 (2014).

8. H. Frei, L. Fredin, G. C. Pimentel, J. Chem. Phys. 74, 397-411 (1981).

9. A. Sinha, M. C. Hsiao, F. F. Crim, J. Chem. Phys. 94, 4928-4935 (1991).

10. R. N. Zare, Science. 279, 1875-1879 (1998).

11. F. F. Crim, Acc. Chem. Res. 32, 877-884 (1999).

12. S. Yan, Y.-T. Wu, B. Zhang, X.-F. Yue, K. Liu, Science. 316, 1723-1726 (2007).

13. D. R. Killelea, V. L. Campbell, N. S. Shuman, A. L. Utz, Science. 319, 790-793 (2008).

14. F. F. Crim, Proc. Natl. Acad. Sci. USA. 105, 12654-12661 (2008).

15. W. Zhang, H. Kawamata, K. Liu, Science. 325, 303-306 (2009).

16. J. C. Polanyi, Acc. Chem. Res. 5, 161-168 (1972).

17. K. Liu, Annu. Rev. Phys. Chem. 67, 91-111 (2016).

18. A. Shalabney et al., Nat. Commun. 6, 5981 (2015). 
19. J. George, A. Shalabney, J. A. Hutchison, C. Genet, T. W. Ebbesen, J. Phys. Chem. Lett. 6, 1027-1031 (2015).

20. J. George et al., Phys. Rev. Lett. 117, 153601 (2016).

21. R. M. A. Vergauwe et al., J. Phys. Chem. Lett. 7, 4159-4164 (2016).

22. A. Thomas et al., Angew. Chem. Int. Ed. 55, 11462-11466 (2016).

23. T. W. Ebbesen, Acc. Chem. Res. 49, 2403-2412 (2016).

24. J. A. Hutchison et al., Adv. Mater. 25, 2481-2485 (2013).

25. S. Wang et al., Nanoscale. 6, 7243-7248 (2014).

26. E. Orgiu et al., Nat. Mater. 14, 1123-1129 (2015).

27. D. M. Coles et al., Nat. Mater. 13, 712-719 (2014).

28. X. Zhong et al., Angew. Chem. Int. Ed. 55, 6202-6206 (2016).

29. X. Zhong et al., Angew. Chem. Int. Ed. 56, 9034-9038 (2017).

30. K. Stranius, M. Hertzog, K. Börjesson, Nat. Commun. 9, 2273 (2018).

31. G. C. Schatz, M. C. Colton, J. L. Grant, J. Phys. Chem. 88, 2971-2977 (1984).

32. A. Grill, D. A. Neumayer, J. Appl. Phys. 94, 6697-6707 (2003).

33. M. Kasha, Radiation Research. 20, 55-70 (1963).

34. J. A. Hutchison, T. Schwartz, C. Genet, E. Devaux, T. W. Ebbesen, Angew. Chem. Int. Ed. 51, 1592-1596 (2012).

35. J. Galego, F. J. Garcia-Vidal, J. Feist, Nat. Commun. 7, 13841 (2016).

36. J. Galego, F. J. Garcia-Vidal, J. Feist, Phys. Rev. Lett. 119, 136001 (2017).

37. S. Gambino et al., ACS Photonics. 1, 1042-1048 (2014).

38. J. P. Long, B. S. Simpkins, ACS Photonics. 2, 130-136 (2015).

39. W. Ahn, I. Vurgaftman, A. D. Dunkelberger, J. C. Owrutsky, B. S. Simpkins, ACS Photonics. 5, 158-166 (2018).

40. M. Hertzog et al., Chem. Eur. J. 23, 18166-18170 (2017).

41. O. Kapon, R. Yitzhari, A. Palatnik, Y. R. Tischler, J. Phys. Chem. C. 121, 18845-18853 (2017).

42. S. R. Casey, J. R. Sparks, J. Phys. Chem. C. 120, 28138-28143 (2016).

43. V. F. Crum, S. R. Casey, J. R. Sparks, Phys. Chem. Chem. Phys. 20, 850-857 (2018).

44. G. G. Rozenman, K. Akulov, A. Golombek, T. Schwartz, ACS Photonics. 5, 105-110 (2018). 
45. D. S. Dovzhenko, S. V. Ryabchuk, Y. P. Rakovich, I. R. Nabiev, Nanoscale. 10, 35893605 (2018).

46. D. G. Baranov, M. Wersäll, J. Cuadra, T. J. Antosiewicz, T. Shegai, ACS Photonics. 5, 24 42 (2018).

47. F. Herrera, F. C. Spano, Phys. Rev. Lett. 116, 238301 (2016).

48. L. A. Martínez-Martínez, R. F. Ribeiro, J. Campos-González-Angulo, J. Yuen-Zhou, ACS Photonics. 5, 167-176 (2018).

49. J. Flick, C. Schäfer, M. Ruggenthaler, H. Appel, A. Rubio, ACS Photonics. 5, 992-1005 (2018).

\section{Acknowledgments:}

Funding: We acknowledge support of the International Center for Frontier Research in Chemistry (icFRC, Strasbourg), the ANR Equipex Union (ANR-10-EQPX-52-01), the Labex NIE projects (ANR-11-LABX-0058 NIE) and CSC (ANR-10-LABX-0026 CSC) within the Investissement d'Avenir program ANR-10-IDEX-0002-02.

Competing interests: Authors declare no competing interests

Data and materials availability: All data is available in the main text or the supplementary materials.

\section{Supplementary Materials:}

Materials and Methods

Figures S1-S6 


\section{Supplementary Materials for}

\section{Tilting a Ground State Reactivity Landscape by Vibrational Strong Coupling}

A. Thomas ${ }^{1 \#}$, L. Lethuillier-Karl ${ }^{1 \#}$, K. Nagarajan ${ }^{1}$, R. M. A. Vergauwe ${ }^{1}$, J. George ${ }^{1 \ddagger}, T$. Chervy $^{1 \dagger}$, A. Shalabney ${ }^{2}$, E. Devaux ${ }^{1}$, C. Genet ${ }^{1}$, J. Moran $^{1 *}$, T. W. Ebbesen ${ }^{1 *}$

${ }^{1}$ University of Strasbourg, CNRS, ISIS \& icFRC, 8 allée G. Monge, 67000 Strasbourg, France.

${ }^{2}$ Braude College, Snunit Street 51, Karmiel 2161002, Israel

10

*Correspondence to: ebbesen@unistra.fr; moran@unistra.fr

\#These authors contributed equally

Present Address:

†Department of Chemistry. Indian Institute of Science Education and research Mohali, Mohali, India.

†Department of Physics, ETH Zurich, Switzerland

This PDF file includes:

20

Materials and Methods

Figs. S1 to S6

\section{Materials and Methods}

Unless otherwise noted, all reagents and solvents were purchased from commercial suppliers and used without further purification.

The IR transmission spectra of the Fabry-Pérot cavities and the 'outside the cavity' cells were acquired by standard FT-IR (Fourier transform infrared) spectrometer (Bruker Vertex70) in transmission mode. The transmission was recorded by DTGS (deuterated triglycine sulfate) detector with $2 \mathrm{~cm}^{-1}$ resolution over 64 scans.

GC-MS analysis was performed on a GC System 7820A (G4320) connected to a MSD block 5977E (G7036A), using Agilent High Resolution Gas Chromatography Column: PN 19091S - 433UI, HP - 5MS UI, $28 \mathrm{~m} \times 0.250 \mathrm{~mm}, 0.25$ Micron, SN USD 489634H. All samples were prepared in 2-propanol (200 $\mu \mathrm{L}$ sample volume). The analysis was carried out on a splitless $1 \mu \mathrm{L}$ injection volume with an injection port temperature 250 ${ }^{\circ} \mathrm{C}$. Column oven temperature program was as follows: $60{ }^{\circ} \mathrm{C}$ for $1 \mathrm{~min}$, ramped at $30^{\circ} \mathrm{C} \mathrm{min}^{-1}$ to $310{ }^{\circ} \mathrm{C}$ with $3 \mathrm{~min}$ hold, with a total running time of $12.33 \mathrm{~min}$. The mass spectrometer was turned on after $2 \mathrm{~min}$ and was operated at 
the electron ionization mode with quadrupole temperature of $150{ }^{\circ} \mathrm{C}$. Data acquisition was performed in the fullscan mode (50-500). Hydrogen (99.999\% purity) was used as carrier gas at a constant flow rate of $1.5 \mathrm{~mL} \mathrm{~min}^{-1}$.

${ }^{1} \mathrm{H}$ NMR spectrum of starting material $\mathbf{L} 1$ in $\mathrm{CDCl}_{3}$ was recorded on a Bruker Avance 400 (400 MHz) spectrometer at ambient temperature and is reported in ppm scale using the residual solvent resonance as the internal reference $\left(\mathrm{CHCl}_{3}\right.$ at $7.26 \mathrm{ppm}$ ). Coupling constants $J$ are reported in $\mathrm{Hz}$, as determined using the software (NMR Notebook v2.80). ${ }^{13} \mathrm{C}$ NMR spectrum was recorded on a Bruker Avance 400 (100 MHz) spectrometer and is reported in ppm using the residual solvent resonance as the internal standard $\left(\mathrm{CDCl}_{3}\right.$ at $\left.77.0 \mathrm{ppm}\right)$.

\section{Synthesis of tert-butyldimethyl((4-(trimethylsilyl)but-3-yn-1-yl)oxy)silane (L1)}

To a stirred solution of 4-(trimethylsilyl)but-3-yn-1-ol (3.50 mmol, 0.585 mL) in DMF (5 $\mathrm{mL})$ at $0{ }^{\circ} \mathrm{C}$ were added imidazole $(8.75 \mathrm{mmol}, 0.596 \mathrm{~g})$ and tert-butylchlorodimethylsilane (4.38 mmol, $0.658 \mathrm{mg})$. The mixture was allowed to warm to room temperature and was stirred for 24 hours. The reaction was quenched by the addition of aq. saturated $\mathrm{NH}_{4} \mathrm{Cl}(15 \mathrm{~mL})$. The organic layer was collected and extracted with diethyl ether through repeated washings. The combined organic fractions were then washed with water, dried over $\mathrm{Na}_{2} \mathrm{SO}_{4}$ and concentrated under reduced pressure to give the crude product as a pale yellow liquid. The crude product was purified by column chromatography on silica using a mixture of ethyl acetate and petroleum ether $(1: 9 ; \mathrm{v} / \mathrm{v})$ as the eluent to give the pure product L1 $(0.754 \mathrm{~g} ; 84 \%)$ as a colourless liquid.

${ }^{1} \mathbf{H}$ NMR $\left(400 \mathrm{MHz}_{\mathrm{CDCl}}\right) \delta 3.72(\mathrm{t}, J=7.0 \mathrm{~Hz}, 2 \mathrm{H}, \mathbf{3}), 2.44(\mathrm{t}, \mathrm{J}=7.0 \mathrm{~Hz}, 2 \mathrm{H}, 4), 0.90(\mathrm{~s}, 9 \mathrm{H}$, 1), $0.14(\mathrm{~s}, 9 \mathrm{H}, \mathbf{5}), 0.08(\mathrm{~s}, 6 \mathrm{H}, 2)$

${ }^{13}$ C NMR (101 MHz, $\left.\mathrm{CDCl}_{3}\right) \delta 104.29$ (F), 85.61 (G), 61.86 (D), 25.93 (A), 24.31 (B), 18.40 (E), $0.12(\mathbf{H}),-5.22$ (C) 

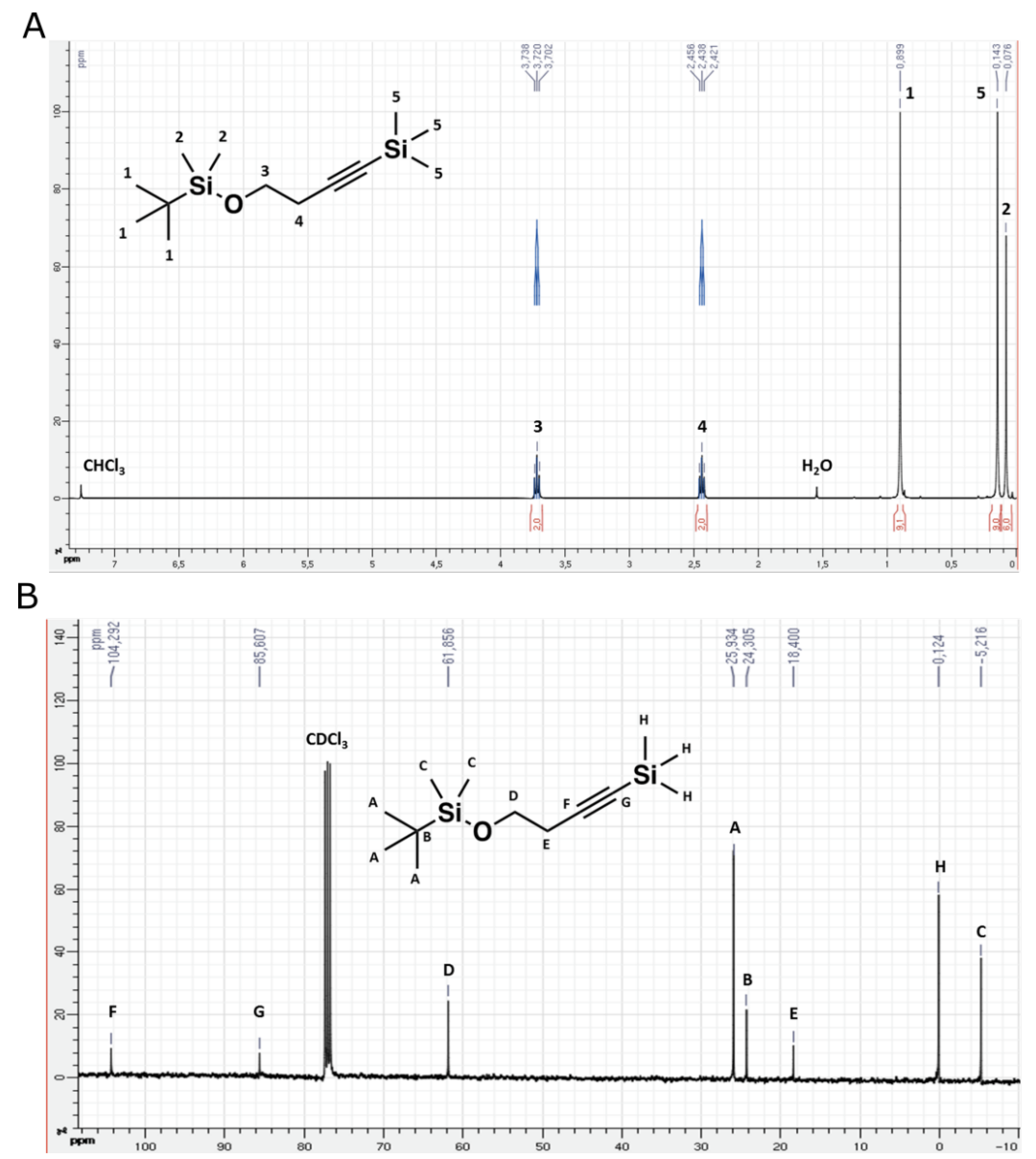

Fig. S1. (A) shows the ${ }^{1} \mathrm{H}$ NMR spectrum of the reactant $\mathrm{L} 1$ with its various ${ }^{1} \mathrm{H}$ signals labelled from 1 to 5 on the schematic structure of $\mathrm{L} 1$ given in the inset. (B) Shows the ${ }^{13} \mathrm{C}$ NMR spectrum with its various ${ }^{13} \mathrm{C}$ signals marked from A to $\mathrm{H}$.

\section{Preparation of the Fabry-Pérot cavity}

The IR cell (demountable flow cell) compatible for temperature controlled measurements was purchased from Specac. The ZnSe $(2 \mathrm{~mm})$ were used as the IR-transmitting window substrates. The metal mirrors for the Fabry-Pérot cavity were prepared by evaporating $\mathrm{Au}(10 \mathrm{~nm})$ on to the $\mathrm{ZnSe}$ windows. To ensure chemical insulation during the reaction from both the metal and $\mathrm{ZnSe}$, each windows were coated with $200 \mathrm{~nm}$ of glass. To obtain the Fabry-Pérot cavity, the Au coated windows were placed in the IR cell, sandwiched by Mylar spacers ( $\sim 6$ $12 \mu \mathrm{m})$ and tightened with the screws. The on- as well as off- resonance cavities were prepared by varying the spacer thickness.

For reference experiments, mentioned as outside the cavity, the liquid cells were prepared in a similar manner as explained above but replacing the metal mirror with glass $(200 \mathrm{~nm})$ coated $\mathrm{ZnSe}$ windows.

Tuning the Fabry-Pérot cavities:

Once an air-filled Fabry-Pérot cavity (hereafter referred as "empty" cavity) is prepared as mentioned above, 
such an "empty" cavity of length L, will show a transmission spectrum with a series of resonances. The frequency of these modes, which are multiples of the fundamental cavity mode, in wavenumber units is given by the equation

$$
\bar{v}_{\left(\mathrm{cm}^{-1}\right)}=\frac{10^{4} \mathrm{~m}}{2 n L}
$$

where $\mathrm{m}$ is the mode order, $\mathrm{n}$ is the refractive index of the medium and $\mathrm{L}$ is the thickness of the cavity in micrometer. The spacing between each mode, called the free spectral range (FSR), gives the frequency corresponding to the fundamental mode. For a given spacer thickness and metal mirrors, the FSR of such an "empty" cavity will be a constant and the injection of the sample will further change the cavity mode spacing as a function of the refractive index $n$ of the injected sample. As a general procedure, to prepare an on-resonance cavity with respect to a given vibrational transition, we first calculate the refractive index $n$ of the medium to be injected. Subsequently, the "empty" cavity is tuned to get the required the FSR, such that when the sample is injected, one of the cavity mode is in resonance with the chosen vibrational transition. For example, in the present experiments, to make an on-resonance cavity with Si-C stretching transition at $842 \mathrm{~cm}^{-1}$, we first tuned our "empty" cavity to attain the FSR of $586 \mathrm{~cm}^{-1}$ as shown in Fig. S2A. Therefore the injection of our reaction mixture, whose $\mathrm{n}$ is 1.395 , will modify the FSR to $420 \mathrm{~cm}^{-1}$ and result in the strong coupling of the Si-C stretching transition with the second mode of the cavity as shown in Fig. S2b.

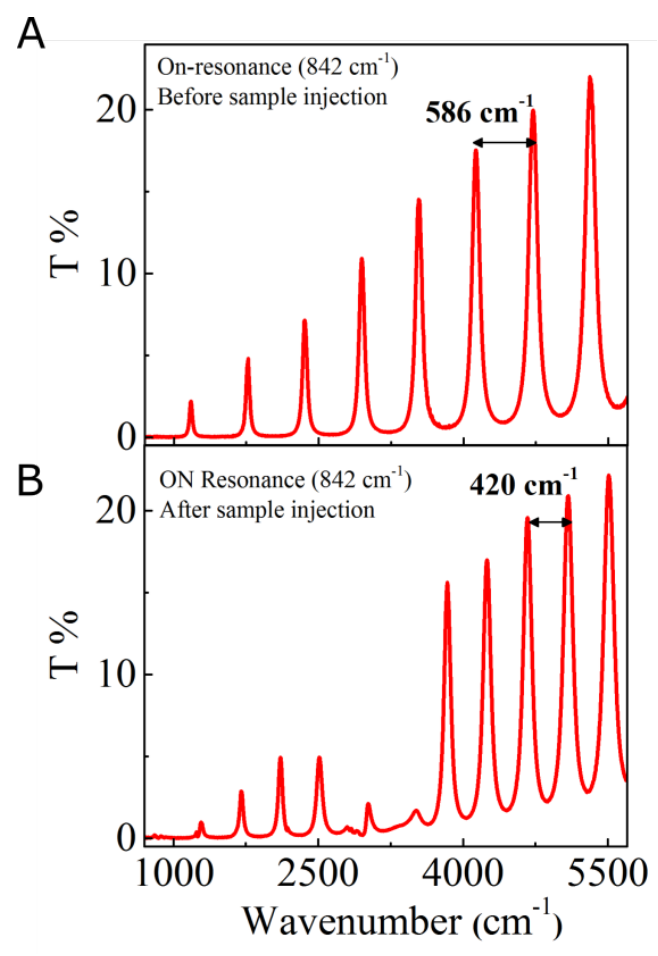

Fig. S2. Measured optical resonances of a microfluidic Fabry-Pérot cavity of length $8.5 \mu \mathrm{m}$ before (A) and after (B) the injection of reaction mixture 


\section{Preparation of the reaction mixture and injection into the Fabry-Pérot cavities}

Once the desired "empty" cavity was obtained, the liquid sample cell was inserted in the variable temperature mount and was kept for 2 hours to stabilise and equilibrate to the experimental temperature $\left(25^{\circ} \mathrm{C}\right.$, except if noted otherwise).

The sample injection into the cavities and liquid cells were done as follows. A stock solution of TBAF (3 M) was prepared using a mixture of $\mathrm{MeOH}$ and $\mathrm{THF}$ in 1:1 ratio $(\mathrm{v} / \mathrm{v})$. For the reaction medium, the starting material $\mathrm{L} 1(0.31 \mathrm{mmol}, 100 \mu \mathrm{L})$ was mixed $\mathrm{MeOH}(75 \mu \mathrm{L})$ and THF $(75 \mu \mathrm{L})$ in a $5 \mathrm{~mL}$ glass vial followed by the addition of TBAF stock solution $(100 \mu \mathrm{L})$. The mixture was shaken to obtain a homogeneous solution and was then directly injected into the microfluidic Fabry-Pérot cavity making sure that the cavity is filled without any airbubbles.

\section{Rate of the reaction}

The rate of the reaction was calculated from the shift in the higher order cavity mode. The reactant and the products have slightly different reactive indices resulting in a change of the background refractive index (n) of the reactive medium as the reaction progresses. It will lead to shift of the cavity modes, as evident from Eq. S1. The use of $\mathrm{ZnSe}$ windows for making the sample cells for outside the cavity experiments resulted in a very weak interference fringes as shown in Fig. S3C. This is due to the relatively large difference between the $\mathrm{n}$ of $\mathrm{ZnSe}$ and the reactive medium. These weak interference fringes also shifted in the same was as that of the cavity mode, indicating the progress of the reaction.

To calculate the rate of the reaction, the temporal shift $\left(\Delta \bar{v}_{t}\right)$ of a higher order cavity mode peak for all onas well as off-resonance experiments with respect to the starting value $\left(v_{0}\right)$ was recorded. For experiments outside the cavity, the peak of a weak interference fringe was considered. For all these experiments a mode at $\sim 5000 \mathrm{~cm}^{-1}$, well isolated from the molecular vibrational modes were monitored as shown in Fig.S3. The maximum shift $\left(\Delta \bar{v}_{\infty}\right)$ corresponding to the completion of the reaction was determined separately from an outside the cavity experiment. The $\ln$ plot of the temporal shift $\left(\ln \left(1-\Delta \bar{v}_{t} / \Delta \bar{v}_{\infty}\right)\right)$ gave a straight line, indicating the first order reaction and from the slope the rate constant for the total reaction $\left(k_{T R}\right)$ was calculated. 

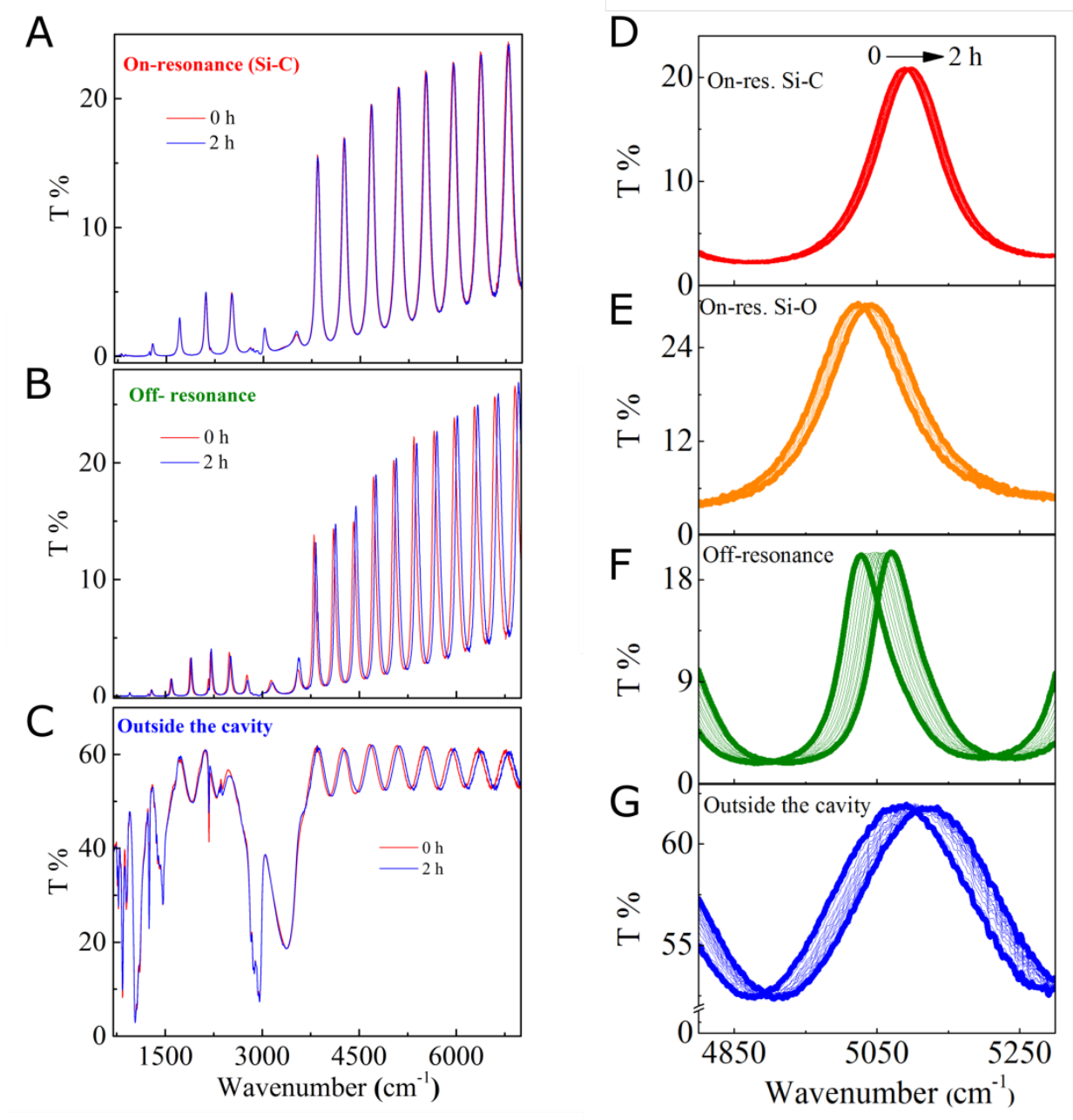

Fig. S3. FT-IR transmission spectrum of the on-resonance cavity (A), off-resonance cavity (B), and outside the cavity (C) immediately after injection of the reaction mixture (red curve) and after $2 \mathrm{~h}$ (blue curve). (D-G) Shows the temporal shift of the higher cavity order mode during the reaction ( 0 to $2 \mathrm{~h}$ ) for cavities on-resonance with the Si-C (D) and the Si-O stretching mode (E), off-resonance cavity (F) and outside the cavity (G). 


\section{Products identification and quantitative GC-MS analysis}

Reaction products $\mathbf{1}$ and $\mathbf{2}$ were identified by comparing the mass spectra and retention times against commercially available samples of $\mathbf{1}$ and $\mathbf{2}$ (Fig. S4)

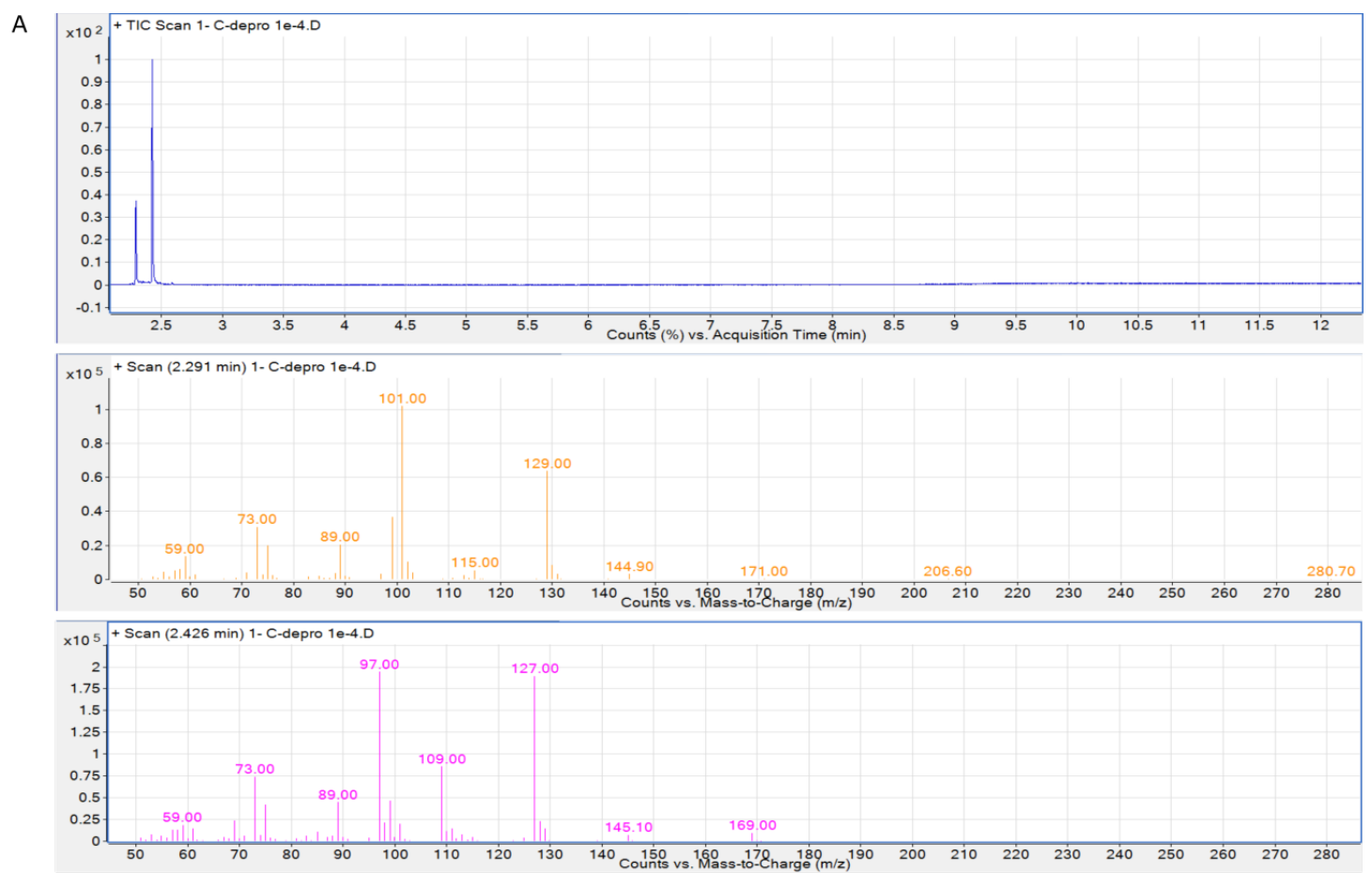

B
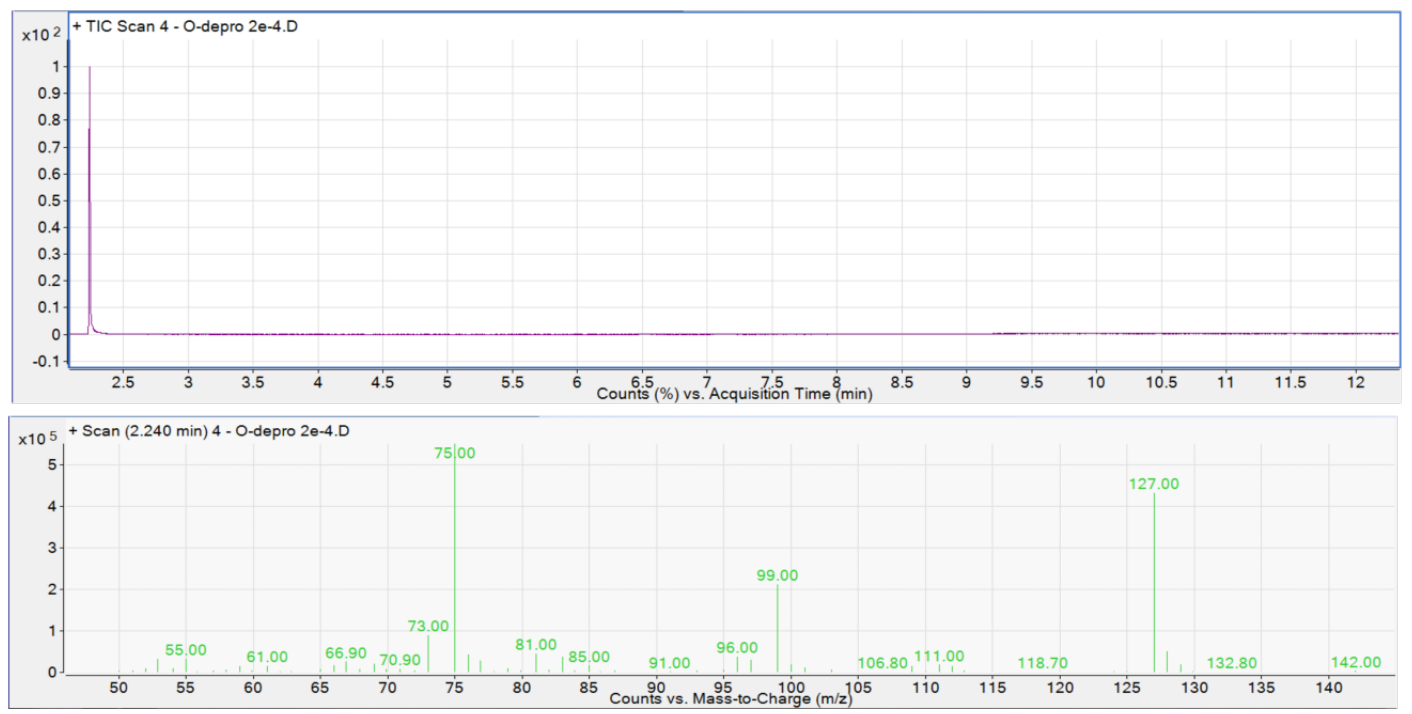

Fig. S4. GC trace and mass spectra of commercially available product $\mathbf{1}$ (A) and 2 (B) 


\section{GC-MS calibration curves and the analysis of the products}

$200 \mu \mathrm{L}$ solutions of compound 1 and 2 at different concentrations (0.1 to $10 \mathrm{mM})$ were prepared by diluting stock solutions of these compounds in isopropanol. For each compound, a six-point graph was plotted; correlating the characteristic GC peaks (as integrated automatically by the Agilent MassHunter Workstation v.B.06.00 software) with substrate concentration (Fig. S5). Each data point was the mean of three independent measurements and the correlation line was obtained from the least-squares fitting (intercept $=0$ ). Error bars on graphs are shown as \pm standard deviation for each data point.

A

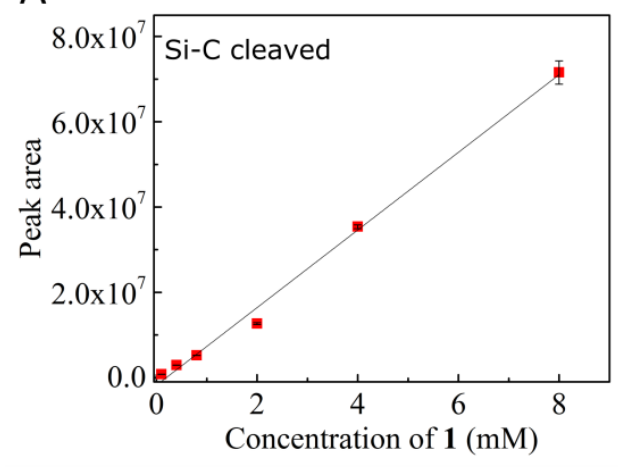

B

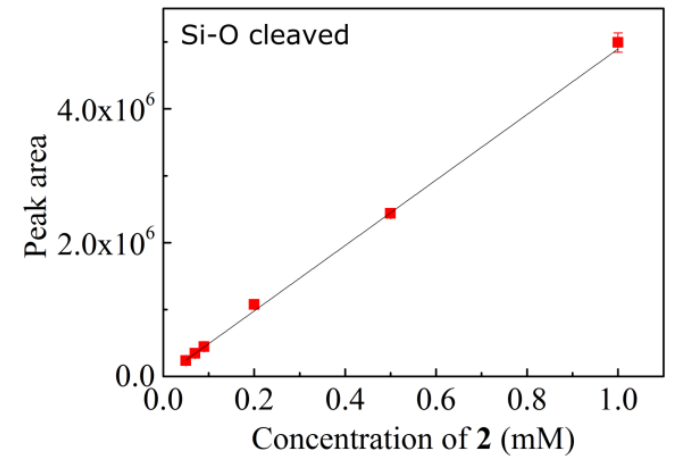

Fig. S5. Correlation between the concentration of the products in isopropanol and the measured gas chromatography peak areas. The calibration curve for $\mathrm{C}$ - and O-deprotected products are shown in (A) and (B) respectively.

After $3 \mathrm{~h}$ of reaction, the microfluidic Fabry-Pérot cavity (for on- and off-resonance measurements) as well as the liquid cells of the outside the cavity experiments was opened and the reaction mixture was extracted with isopropanol $(1 \mathrm{~mL}) .200 \mu \mathrm{L}$ of this solution were directly injected in GC-MS. The concentrations of 1 and 2 in the reaction mixture were calculated by comparing the product peak area with the calibration line and the ratio between the two products (1 over $\mathbf{2}$ ) was determined. The reported ratios are an average of multiple runs, with an error corresponding to the mean $( \pm)$ absolute deviation. 


\section{Kinetics of reactions strongly coupled to the C-O stretching vibration of $\mathrm{L} 1$ and solvents}
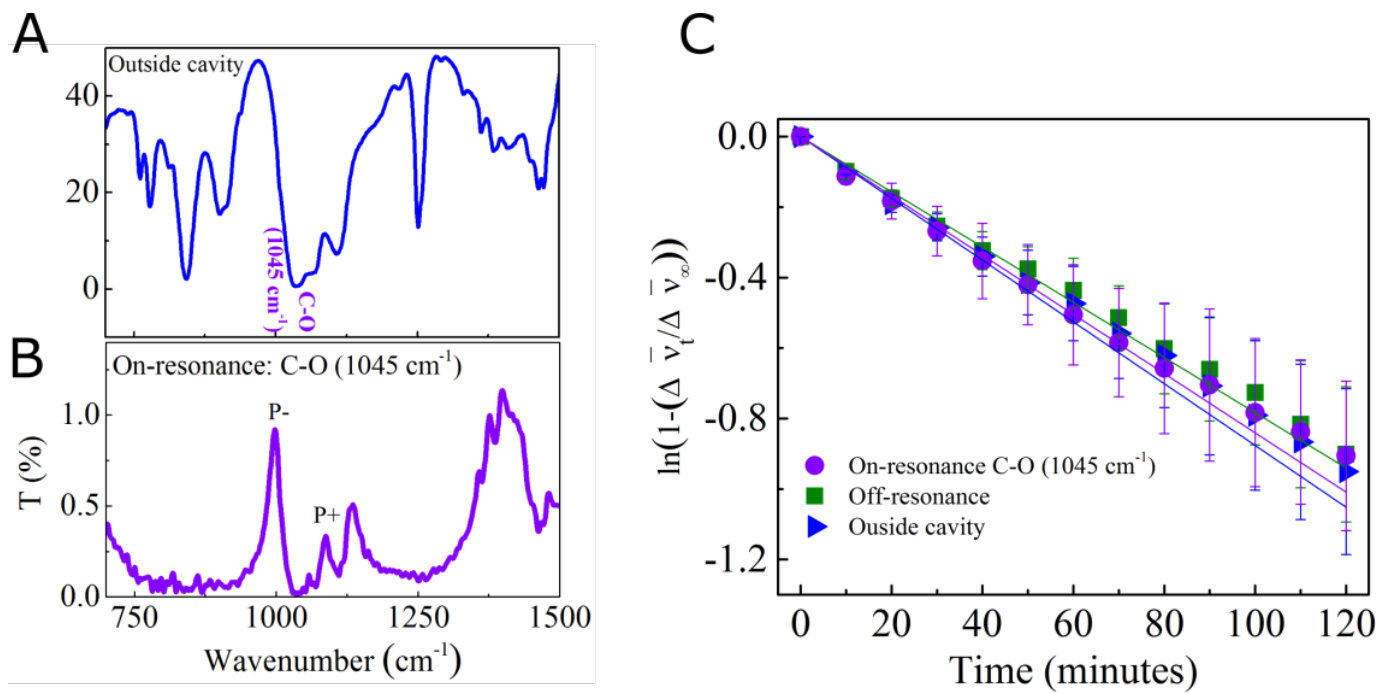

5

Fig. S6. (A) FT-IR transmission spectrum of the starting material L1 in the reaction mixture consisting of $\mathrm{MeOH}$ and THF, recorded outside cavity with the $\mathrm{C}-\mathrm{O}$ stretching vibration noted in the plot. (B) The FT-IR transmission spectra showing the vibrational strong coupling of the $\mathrm{C}-\mathrm{O}$ stretching vibration of $\mathrm{L} 1, \mathrm{MeOH}$ and THF. (C) Kinetics of the reactions in cavities tuned to be on-resonance with the $\mathrm{C}-\mathrm{O}$ stretching mode of L1 (violet circles) together with an off-resonance cavity (green squares) and outside the cavity (blue triangles). 
